\title{
Inclusion of Trithia[5]heterohelicene by Various Serum Albumins. An Effective Probe for Chiral Discrimination
}

\author{
Koh-ichi Yamada, ${ }^{*}$ Rieko Ishil, Hiroko Nakagawa, and Hiroshi Kawazura \\ Faculty of Pharmaceutical Sciences, Josai University, Sakado, Saitama 350-02, Japan. \\ Received December 12, 1996; accepted February 6, 1997
}

\begin{abstract}
Racemic 2-hydroxymethyltrithia[5]heterohelicene (2-HT), which has a labile helical structure was incorporated into serum albumins of nine species in 1\% ethanolic aqueous solution, giving $1: 1$ complexes which exhibited induced circular dichroism spectra with species specificity. The application of 2-HT to bovine serum as a probe for chiral recognition revealed that the serum itself manifested an apparent chiral discrimination between enantiomers of 2-HT.
\end{abstract}

Key words thiaheterohelicene; induced circular dichroism; serum albumin; chiral discrimination; species specificity

In organic and bioorganic chemistry, induced circular dichroism (ICD) can be reliably used for the evaluation of host-guest interactions and non-covalent binding forces, such as those involved in substrate specificity and binding affinity. ${ }^{1)}$ Many of these studies have been carried out by using kinetically labile racemates, where the asymmetric environment may be capable of performing chiral discrimination between enantiomers. ${ }^{2)}$ These ICD techniques occasionally encounter the problem that the observed spectra are difficult to interpret when racemates having only weakly absorbing chromophores are utilized. Recently, we found that racemic 2-hydroxymethylthieno[3,2-e:4,5-e'] di[1]benzothiophene (2-hydroxymethyltrithia[5]heterohelicene, 2-HT), which racemizes rapidly at room temperature, ${ }^{3)}$ was converted into the $P$ enantiomer upon uptake by bovine serum albumin (BSA) in an aqueous solution, exhibiting intense ICD bands in the region of the 2-HT absorptional wavelengths. ${ }^{4}$ ) This phenomenon of a sort of second-order asymmetric transformation $^{5)}$ suggested that 2-HT may be useful as a potential probe for assessing the ability of albumins for chiral discrimination between enantiomers, because of the ease of assignment of the ICD absorptions due to the $P$ and $M$ conformations of 2-HT. In this paper we describe chiral discrimination by serum albumins of different species and by serum itself between the enantiomers of 2-HT, as studied by ICD.

An equimolar amount of racemic 2-HT in ethanol was added to an aqueous solution of serum albumin and the mixture was incubated at $25 \pm 1{ }^{\circ} \mathrm{C}$ to give a transparent solution in $1 \%$ ethanol-water. Figure 1 shows the UV spectra of the solutions containing $1: 1$ complexes between various serum albumins and 2-HT. Every spectrum has two intense absorption maxima around 333 and $348 \mathrm{~nm}$, demonstrating no significant overall species-difference in the absorptional energies and intensities. None of the

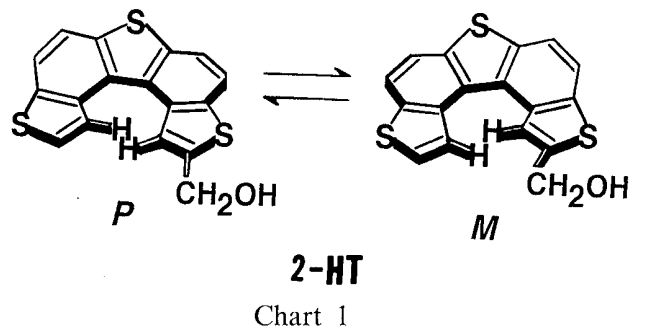

* To whom correspondence should be addressed. serum albumins investigated here possesses any absorption peak in the range over $300 \mathrm{~nm}$ in aqueous solution. Therefore, the peaks observed in the spectra are due to the incorporated 2-HT molecules, which have a strongly absorbing chromophore.

In marked contrast to the slight differences, if any, in the UV spectra, there were marked distinctions between the shapes and intensities of the ICD spectra measured for the nine complexes (Fig. 2). In particular, striking features appear in the longer-wavelength region $(280-$ $375 \mathrm{~nm}$ ) where no serum albumin studied has a CD absorption maximum. In every ICD spectrum, three absorption bands due to the 2-HT molecule, i.e., $\alpha$-, $p$ - and $\beta$-bands (Fig. 2), which are characteristic of condensed aromatics and the helicene family, ${ }^{6}$ ) can be recognized. The signs of the Cotton effects for the corresponding bands in each spectrum proved to be the same; this situation may be easily understood by inspection of the negative $\alpha$-band of the longest wavelength. It has already been confirmed that the $P$ enantiomers of 2-HT and its congener thiaheterohelicenes ${ }^{6,7)}$ show a negative sign for

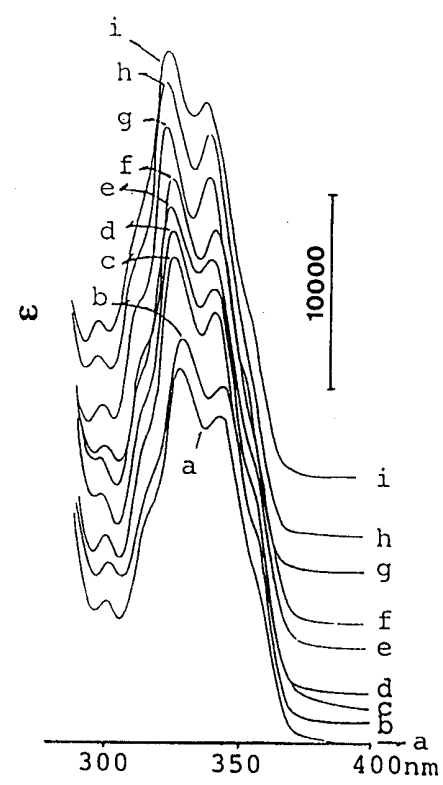

Fig. 1. UV Spectra of $1: 1$ Complexes between Various Serum Albumins and $2-\mathrm{HT}$ in $1 \% \mathrm{EtOH}-$ Water

a, bovine; b, horse; c, human; d, goat; e, pig; f, chicken; g, guinea pig; h, rabbit; i, rat.

(C) 1997 Pharmaceutical Society of Japan 

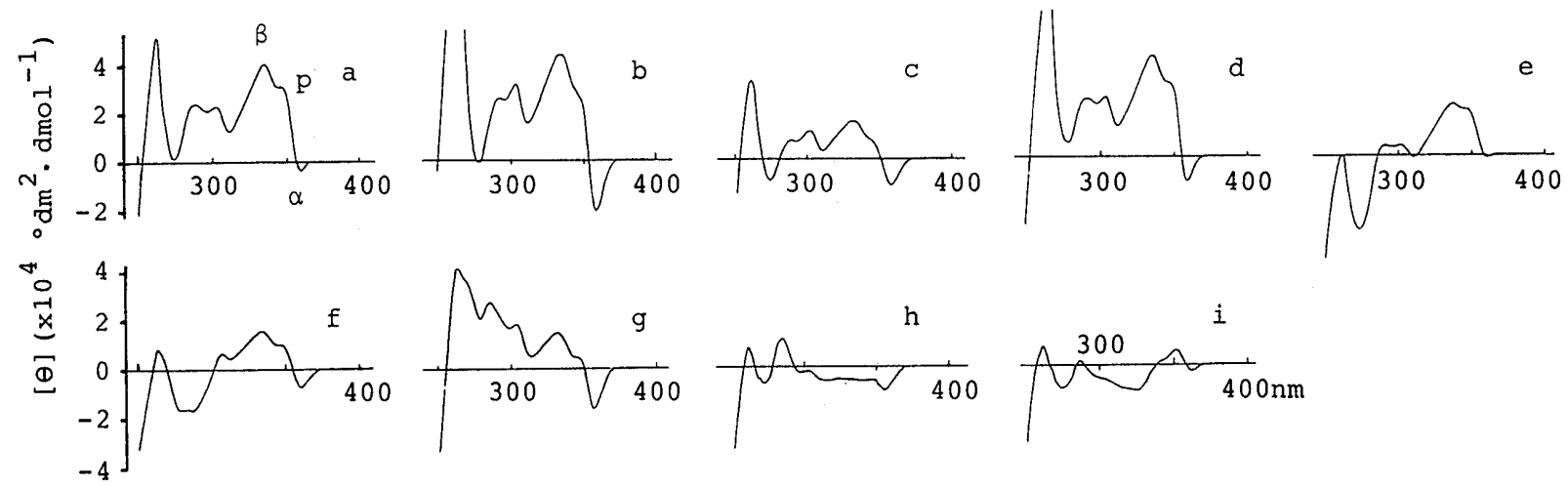

Fig. 2. ICD Spectra of 1:1 Complexes between Various Serum Albumins and 2-HT in 1\% EtOH-Water

a, bovine; b, horse; c, human; d, goat; e, pig; f, chicken; g, guinea pig; h, rabbit; i, rat.

the $\alpha$-band and positive sign for the $p$ - and $\beta$-bands. Consequently, it appears that all the albumins include at least an excess of the $P$ enantiomer over its antipode.

It is reasonable to expect that these species-differences in the shapes and magnitudes of the ICD bands should arise from differences in the spatial structure of the 2-HT uptake site of the albumins. Structural analyses of $\mathbf{B S A}^{8)}$ as well as human serum albumin, ${ }^{9)}$ have revealed three uptake sites for substrates, of which two are utilized for accommodation of 2-HT in the case of BSA. ${ }^{4}$ Although we have not yet identified which site are capable of incorporating the 2-HT molecules in the various serum albumins studied, the following scenario is plausible. The serum albumins of bovine, horse, human, goat and pig are anticipated to possess 2-HT uptake sites of similar shape and size, because of the similarity of their ICD spectra. On the other hand, the 2-HT uptake sites of rabbit, rat, and guinea pig serum albumins are presumed to have a considerably different spatial structure from that of BSA. Furthermore, though the complexes contain the same amount of 2-HT, the apparent ellipticities vary widely among them, possibly reflecting differences in the ability of the serum albumins to recognize the chirality of substrates.

In order to examine further the usefulness of 2-HT as a probe for chiral discrimination, 2-HT was added to bovine serum which was reconstituted by dissolving lyophilized powder in water. The clear solution gave virtually the same UV and ICD absorption patterns (Fig. 3) as those of the BSA-2-HT complex except for slight broadening of the bands. This indicates that the serum itself can apparently show uptake and chirality recognition of $2-\mathrm{HT}$, without interference by the many components other than albumin that normally occur in it. Further, the effectiveness of 2-HT was examined in similar experiments using bovine serum preheated to $50-70^{\circ} \mathrm{C}$. Conformational changes of serum albumins gradually increase with a rise in temperature up to $70^{\circ} \mathrm{C},{ }^{10}$ ) and the ability of the BSA preheated at $70^{\circ} \mathrm{C}$ to recognize chirality was markedly reduced without any significant alteration in 2-HT uptake ability. ${ }^{4}$ Table 1 summarizes the results, which were quite similar to the observations regarding the preheated BSA. Up to the treatment temperature of $70^{\circ} \mathrm{C}$, the $\mathrm{UV}$ and $\mathrm{CD}$ changes of the serum itself seem to be small (right columns in the table). On

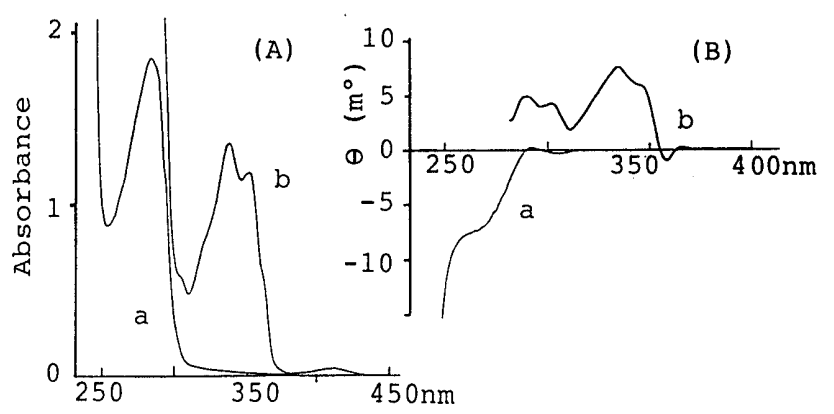

Fig. 3. UV (A) and ICD (B) Spectra of Bovine Serum (a), and Bovine Serum and 2-HT (b)

Table 1. Dependence of UV and CD Intensities of Preheated Bovine Serum and 2-HT upon the Preheating Temperature

\begin{tabular}{cccccc}
\hline \hline $\begin{array}{c}\text { Preheating } \\
\text { temp. } \\
\left({ }^{\circ} \mathrm{C}\right)\end{array}$ & \multicolumn{2}{c}{ Serum $+2-\mathrm{HT}$} & & \multicolumn{2}{c}{ Serum } \\
\cline { 2 - 3 } \cline { 5 - 6 } \cline { 5 - 6 } & $\mathrm{UV}(\%)^{a)}$ & $\mathrm{CD}(\%)^{b)}$ & & $\mathrm{UV}(\%)^{c)}$ & $\mathrm{CD}(\%)^{d)}$ \\
\hline 50 & 99 & 87 & & 99 & 96 \\
60 & 98 & 48 & & 101 & 97 \\
70 & 98 & 26 & & 104 & 82 \\
\hline
\end{tabular}

The preheating time was $1 \mathrm{~h}$ and the intensities using intact bovine serum were taken as $100 \%$. Obtained from $a$ ) $\varepsilon(348 \mathrm{~nm}), b$ ) $\theta$ (peak at $333 \mathrm{~nm})-\theta$ (through at $310 \mathrm{~nm}), c) \varepsilon(278 \mathrm{~nm})$, and $d) \theta(270 \mathrm{~nm})$.

the other hand, the ability of the serum to discriminate between enantiomers was found to decrease to a large extent (CD changes), though the ability to incorporate 2-HT remained almost unaltered (UV changes).

The results described herein indicate that the mobile configurations of 2-HT respond sensitively to changes in the chiral environment in which the compound is included. This interesting attribute along with the large molecular ellipticity suggests that 2-HT will be useful as a probe for chiral discrimination.

\section{Experimental}

CD spectra were measured on a Jasco J-20A spectropolarimeter and UV spectra were obtained with a Hitachi UV220 spectrophotometer. Racemic 2-hydroxymethylthieno-[3,2-e:4,5-e']di[1]benzothiophene (2HT) was prepared as described.4) Nine sorts of serum albumins (essentially fatty acid-free reagents) examined in this study were purchased from Sigma. Bovine serum was obtained by dissolving lyophilized powder in water. Water used in the experiments was distilled and then passed through a Millipore Milli-QIII purification system. 
Solutions of 2-HT-Serum Albumin (1:1) Complex The solutions of the $1: 1$ complexes were obtained by mixing $0.1 \mathrm{ml}$ of an ethanolic stock solution $\left(1.53 \times 10^{-3} \mathrm{~mol} \mathrm{dm}^{-3}\right)$ of 2 -HT, which is insoluble in water, with $5 \mathrm{ml}$ of an aqueous solution $\left(3.06 \times 10^{-5} \mathrm{moldm}^{-3}\right)$ of serum albumin, followed by adjustment of the volume to $10 \mathrm{ml}$ with water to give the same concentration of $1.53 \times 10^{-5} \mathrm{~mol} \mathrm{dm}^{-3}$ for both components. The mixture was slightly turbid, but gentle agitation for $30 \mathrm{~min}$ at $25 \pm 1^{\circ} \mathrm{C}$ afforded a transparent solution.

Preparation of 2-HT Solution in Bovine Serum The stock solution of bovine serum was prepared by dissolving the lyophilized serum powder (Sigma, Sera S-1507) in $5 \mathrm{ml}$ of water, and diluting this solution to $100 \mathrm{ml}$ with water. The concentration of this stock solution was about $1 / 20$ of that of bovine serum prior to lyophilization. A 2-HT ethanolic solution $\left(0.1 \mathrm{ml}, 6.13 \times 10^{-3} \mathrm{M}\right)$ was added to $4 \mathrm{ml}$ of the stock solution of the serum, and the volume was adjusted to $10 \mathrm{ml}$ with water. The mixture was incubated for $30 \mathrm{~min}$ at $25 \pm 1{ }^{\circ} \mathrm{C}$ with gentle stirring to give a clear solution.

The preheated serum was obtained by incubating part of the stock solution of the serum for $1 \mathrm{~h}$ at 50,60 , or $70^{\circ} \mathrm{C}$, respectively. The solutions containing the preheated serum and 2-HT were prepared by the same procedures as above.

\section{References}

1) Ahmad H., Snatzke G., Atta-ur-Rahman, J. Am. Chem. Soc., 115,
12533-12544 (1993) and references cited therein; Tokura N., Nagai T., Takenaka S., Oshima T., J. Chem. Soc., Perkin Trans. 2, 1974, $337-342$.

2) Krois D., Lehner H., J. Chem. Soc., Perkin Trans. 2, 1995, 489-494 and references cited therein.

3) Yamada K., Nakagawa H., Kawazura H., Bull. Chem. Soc. Jpn., 59, 2429-2432 (1986).

4) Yamada K., Ishii R., Nakagawa H., Kawazura H., J. Chem. Soc., Chem. Commun., 1994, 1521-1522; idem, Tetrahedron: Asymmetry, 7, 737-746 (1996).

5) Jacques J., Collet A., Wilen S. H., "Enantiomers, Racemates, and Resolutions," Wiley, New York, 1981, pp. 369-377.

6) Groen M. B., Wynberg H., J. Am. Chem. Soc., 93, 2968-2974 (1971); Groen M. B., Stulen G., Visser G. J., Wynberg H., ibid., 92, 7218-7219 (1970); Clar E., Chem. Ber., 69, 607-614 (1936).

7) Yamada K., Tanaka H., Nakagawa H., Ogashiwa S., Kawazura H., Bull. Chem. Soc. Jpn., 55, 500-503 (1982).

8) Luft A. J., Lorscheider F. L., Biochemistry, 22, 5978-5981 (1983).

9) He X. M., Carter D. C., Nature (London), 358, $209-215$ (1992).

10) Lin V. J. C., Koenig J. L., Biopolymer, 15, 203-218 (1976); Wetzel R., Becker M., Behlke J., Billwitz H., Bohm S., Ebert B., Hamann H., Krumbiegel J., Lassmann G., Eur. J. Biochem., 104, 469-478 (1980). 